\title{
Effective attractive polariton-polariton interaction mediated by the exciton reservoir
}

\author{
D. V. Vishnevsky and F. Laussy \\ Condensed Matter Physics Center (IFIMAC), Departamento de Física Teórica de la Materia Condensada, \\ Universidad Autónoma de Madrid, 28049 Madrid, Spain*
}

\begin{abstract}
We present a mechanism to endow the polariton gas with attractive interactions. The scheme relies on an exciton reservoir, which can be formed even if the excitons lie outside of the light-cone. Such an attractive interaction should open new routes in the physics of quantum fluids, that we illustrate with a simple but nontrivial application whereby the polariton condensate enters the selfoscillation regime powered by the attraction from the reservoir. This results in pulsed emission in the $\mathrm{GHz}$ regime.
\end{abstract}

PACS numbers: 71.36.+c, 71.35.Lk, 03.75.Mn

\section{INTRODUCTION.}

Cavity polaritons have attracted the attention of scientists since their discovery over twenty years ago $\frac{1}{\underline{ }}$ for their unique properties as quasi-particles, e.g., extremely small, but nonzero effective mass, interactions, integer spin making them bosons, etc. $\stackrel{2}{2}$ This led to a number of outstanding fundamental phenomena: Bose-Einstein condensation (BEC), superfluidity, Josephson oscillations and many others remarkable properties have been reported recently $\underline{\underline{3}} \underline{\underline{-5}}$. One of the key features of polaritons is their nonlinear optical properties, which opens to them the physics of interacting systems, such as optical parametric oscillations $\underline{\underline{6}}$, bi- and multistability effects $\underline{\underline{7}-\underline{\underline{9}}}$, numerous topological defects $\frac{10}{}$, solitonic physics 11 , etc. Also, boson-boson interaction have an important role in defining the BEC properties. Unlike atoms which have in general attractive interactions, which may result in a collapse of the $\mathrm{BEC} \stackrel{12}{ }$, polaritons have repulsive interactions 13 . Weak attraction can occur between crosspolarized polaritons $\frac{14}{4}$, but this is in addition to the dominant co-polarized repulsion.

It is increasingly appreciated that the polariton gas features one additional strong departure from its atomic counterpart, which is the presence of an exciton reservoir, i.e., particles uncoupled to light that lie at higher energies and higher $k$ vectors, and which provide a supply of particles for the short-lived polaritons at the bottom of their dispersion. The reservoir can extend considerably the lifetime of a polariton experiment $\frac{15}{15}$ and even manifests directly $\underline{16}$. As a rule, it strongly renormalizes the interaction strength, leading to a blueshift of the polariton emission 17,18 . The exciton reservoir arises not only when it is created by direct incoherent excitation, but can also be formed from resonant ground state excitation ${ }^{19,20}$. Several experiments have reported a peculiar dynamics of the polaritons, such as a collapse of the polariton wavepacket $\underline{21}$ or a negative circular polarization of the condensate ${ }^{22}$. Such effects could be understood with an effective attractive interaction $\underline{23}$, but its exact nature is still unknown.

In this paper, we provide a mechanism to bring-in an attractive effective interaction for the polaritons, that is dominant over the other types of interactions. The core idea is to rely on high- $k$ excitons which indeed attract the ground state polaritons through their excitonic component. This should open new directions both to study the fundamental physics of these strongly correlated gas, but also for applications since, thanks to their extremely long lifetime and heaviness, high $k$ excitons can provide engineerable potentials for polaritons, which is one of the prerequisites to use them in logical gates $\underline{24}$. It seems that at the moment there is no way to imprint a dynamical negative potential (potential wells) of arbitrary shape. For instance, due to the repulsive polariton-polariton interactions, optical barriers can provide only positive potential (potential walls). Creation of negative potentials from the attractive interaction between cross-polarized particles has a lot of restrictions as well, due to the instability of the polarization and of the weakness of such an interaction. Acoustic waves 25 brought up considerable tuning but do not allow for structures of arbitrary shapes. By using the long-lived exciton reservoir to create the potential structures, on the other hand, one can imprint virtually any shape from the excitation spot. As one example, we will show here how an attractive exciton reservoir can lead to $\mathrm{GHz}$ self-oscillations in the emission intensity ${ }^{26}$, adding another pulsed emitter in this regime of operation to the few known to date, e.g., with silicon photonic crystal nanocavities 27 .

\section{POLARITON-POLARITON INTERACTIONS.}

Polaritons are composed from photons and excitons and contributions from both fractions should be considered to describe polariton-polariton interaction. However, the main contribution to the energy shift induced by polariton-polariton interaction has been shown experimentally to be brought by their exciton component $\underline{14}$. Theoretical works predict an additional term for the scattering rates due to the exciton oscillator strength saturation that comes from the photonic component ${ }^{13,28}$, that is however small in comparison. It has also been shown that interactions coming from the direct polaritonpolariton scattering is of a repulsive nature, while indi- 
rect scattering processes can be attractive, but again of a smaller magnitude. Indeed, all such scattering processes, except weak Van-der-Waals scatterings, are for polaritons with opposite polarization. The strength of such interactions depends strongly on the detuning between the exciton and photon ${ }^{14,29,30}$, and, in best case, does not exceed the strength of the first order repulsive interaction. For this reason, we will focus on the direct exciton-exciton interaction.

The theory was developed by Ciuti et al., where the exciton-exciton scattering was separated into three types of processes: the direct Coulomb repulsion, the exciton exchange interaction and the carrier (electron and hole) exchange interaction ${ }^{31}$. Considering two excitons with wavevectors $\mathrm{Q}$ and $\mathrm{Q}^{\prime}$ with $\Delta \mathrm{Q}=\mathrm{Q}-\mathrm{Q}^{\prime}$, scattering each in the reciprocal space by the amount $\pm \mathbf{q}$, the Hamiltonian describing each of these interaction processes reads:

$$
H_{i}^{j}(\Delta Q, q, \theta)=\frac{e^{2}}{\varepsilon_{0}} \frac{4}{\pi^{2}} \lambda_{2 D} I_{i}^{j}(\Delta Q, q, \theta),
$$

where $i, j$ label the type of interaction, $\theta$ is the angle between $\Delta \mathbf{Q}$ and $\mathbf{q}, \lambda_{2 D}$ is the exciton Bohr radius and $I_{i}^{j}(\Delta Q, q, \theta)$ some overlap integrals - the only part which varies with the type of interaction. For direct and exciton exchange interactions, these integrals admit an analytical solution according to Ref $\underline{31}$ :

$$
\begin{aligned}
& I_{d i r}(\Delta Q, q, \theta)=\frac{\pi^{3}}{2 q \lambda_{2 D}}\left\{\left[1+\left(\frac{1}{2} \beta_{e} q \lambda_{2 D}\right)^{2}\right]^{-3 / 2}-\left[1+\left(\frac{1}{2} \beta_{h} q \lambda_{2 D}\right)^{2}\right]^{-3 / 2}\right\}, \\
& I_{\text {exch }}^{X}(\Delta Q, q, \theta)=I_{d i r}\left(0, \sqrt{(\Delta Q)^{2}+q^{2}-2 \Delta Q q \cos \theta}, 0\right),
\end{aligned}
$$

where $\beta_{e(h)}=m_{e(h)} /\left(m_{e(h)}+m_{h(e)}\right)$ is the relative mass exchange interaction has a more complicated shape: of the electron (hole). The integral $I_{\text {exch }}^{e(h)}$ for the carrier

$$
\begin{aligned}
& I_{e x c h}^{e, h}(\Delta Q, q, \theta)=\int_{0}^{\infty} d x \int_{0}^{2 \pi} d \theta_{x} \int_{0}^{\infty} d y_{1} \int_{0}^{2 \pi} d \theta_{1} \int_{0}^{\infty} d y_{2} \int_{0}^{2 \pi} d \theta_{2} x y_{1} y_{2} \cos \left\{\Delta Q \lambda_{2 D}\left[\beta_{e, h} x \cos \left(\theta-\theta_{x}\right)+\beta_{e, h} y_{1} \cos \left(\theta-\theta_{1}\right)\right]+\right. \\
& \left.+q \lambda_{2 D}\left[-x \cos \theta_{x}-\beta_{e, h} y_{1} \cos \theta_{1}+\left(1-\beta_{e, h}\right) y_{2} \cos \theta_{2}\right]\right\} \times \\
& \times \exp \left(-\left[\left(y_{2} \cos \theta_{2}-y_{1} \cos \theta_{1}-x \cos \theta_{x}\right)^{2}+\left(y_{2} \sin \theta_{2}-y_{1} \sin \theta_{1}-x \sin \theta_{x}\right)^{2}\right]^{1 / 2}\right) \exp (-x) \times \\
& \times \exp \left(-y_{1}\right) \exp \left(-y_{2}\right)\left\{\frac{1}{\sqrt{y_{1}^{2}+x^{2}+2 y_{1} x \cos \left(\theta_{1}-\theta_{x}\right)}}+\frac{1}{\sqrt{y_{2}^{2}+x^{2}-2 y_{2} x \cos \left(\theta_{2}-\theta_{x}\right)}}-\frac{1}{y_{1}}-\frac{1}{y^{2}}\right\} .
\end{aligned}
$$

Generally, polariton-polariton interaction results in two processes: scattering of polaritons from states $\mathbf{Q}$ and $\mathbf{Q}^{\prime}$ to new states $\mathbf{Q}+\mathbf{q}$ and $\mathbf{Q}^{\prime}-\mathbf{q}$, and an effective renormalization of the polariton energy, that can be considered as a scattering from $\left(\mathbf{Q}, \mathbf{Q}^{\prime}\right)$ to $\left(\mathbf{Q}^{\prime}, \mathbf{Q}\right)$. The latter case corresponds to the matrix elements of interaction at $q=0$.

There are several peculiar properties of the integrals. One is that the integral of the direct Coulomb scattering does not depend on the difference in the excitons wavevectors $\Delta \mathrm{Q}$, but on the scattered wave vector $q$ only. Moreover, it vanishes at zero $q$, so the direct interaction has no influence on the states energy renormalization. The integral of the exciton exchange interaction $I_{\text {exch }}^{X}$ has also a certain symmetry with respect to $\Delta Q$ and $q$, namely, $I_{\text {exch }}^{X}(\Delta Q=0, q, \theta)=I_{\text {exch }}^{X}(\Delta Q, q=0, \theta)$.

Figure 1 shows the magnitude of the three integrals as a function of the momentum difference $\Delta Q$ (interrupted lines), and the total interaction (thick line). While the integral expressions $I_{i}^{j}(\Delta Q, q, \theta)$ have been known for over a decade and used in a large body of works $14,19,32$, the focus has been to the best of our knowledge exclusively on the transmitted wavevector $\mathbf{q}$ and for zero or small exchanged wavevector $\Delta Q$, since it is typically much less than the inverse Bohr radius of the exciton. Furthermore, the strength of the interaction is usually considered equal and repulsive for all polaritons. However, as can be seen on Fig. 1, when $\Delta Q \lambda_{2 D}>2.8$, the interaction changes its sign and becomes attractive. Moreover, the magnitude of the attractive interaction for large exchanged wavevectors is of the same order as the magnitude of repulsion in the common case of small exchanged wavevectors.

For the usual value of a Bohr radius in the range from 10 to $100 \mathrm{~nm}$, the interaction is attractive for wavevectors difference of $\Delta Q>2.8 \times 10^{7} \mathrm{~m}^{-1}$. To provide an attractive interaction between ground-state polaritons 


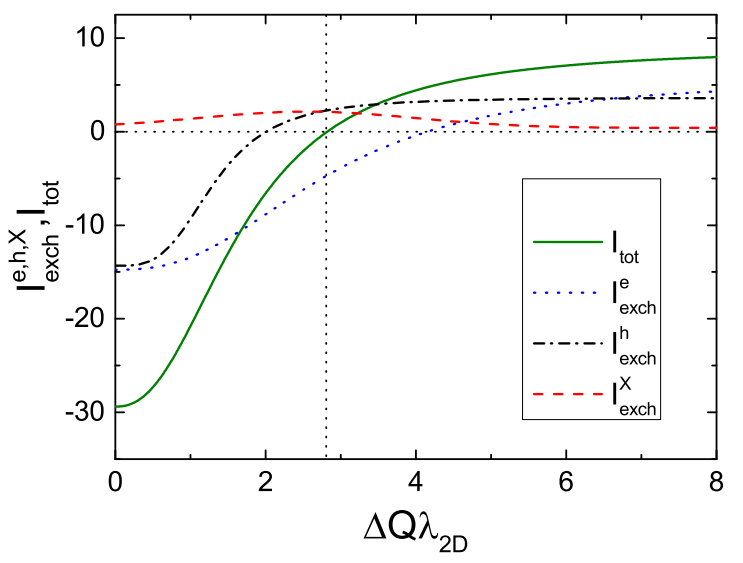

FIG. 1: (Color online) Integrals for electron, hole and exciton exchange interaction (interrupted lines), and their sum (solid line). The vertical dotted line separates the regions of repulsive and attractive interaction, providing the condition $\Delta Q \lambda_{2 D}$ at which the character of the interaction reverses.

and high-energy excitons in GaAs structures, this value of $\Delta Q$ brings the exciton reservoir nearby the frontier of the light-cone, and in common cases it lies outside of it. This means that these excitons are not coupled with light, which can be advantageous to retain them for longer times in their role of an effective reservoir, but also impedes their introduction in the system straightforwardly in an optical way. We therefore propose to use the polariton-polariton scattering in order to create the high$k$ excitons, namely, to scatter off the Upper Polaritons Branch (UPB) with no momentum to the lower branch, with conservation of the energy. Similar processes have already been demonstrated 33 . To estimate the magnitude of attraction that can thus be obtained, let us consider an ordinary cavity with a Rabi splitting of $8 \mathrm{meV}$ at zero detuning. For a typical GaAs exciton mass of $m_{X}=0.5 m_{0}$ and an exciton Bohr radius $\lambda_{2 D}=12.5 \mathrm{~nm}$, the wavevector of the final state is $k_{f}=2.5 \times 10^{8} \mathrm{~m}^{-1}$. This corresponds to the value $\Delta Q \lambda_{2 D}=3$, i.e., the interaction between excitons and polaritons will be well into the attractive regime.

To quantify the interaction strength to scatter polaritons from the bottom of UPB to high- $k$ excitons, one should also calculate Eq. 1 but now taking $\Delta Q=0$. In this case $I_{\text {exch }}^{e}=I_{\text {exch }}^{h}$ and $I_{\text {exch }}^{X}=I_{d i r}$, so only two integrals have to be calculated. Also, since we are interested in the scattering into attractive states, we can assume $q \lambda_{2 D} \geq 2.8$. The result is presented on Fig. 2 Scattering matrix element decreases significantly as scattered wavevector increases. Still, in the region of reciprocal space we are interested in, $I_{t o t}\left(\Delta Q=0, q=k_{f}\right) \approx$ $I_{\text {tot }}\left(\Delta Q=k_{f}, q=0\right)$. In other words, the strength of scattering of two polaritons has the same order as the strength of exciton-polariton interaction.

To describe in more details the mechanism of the

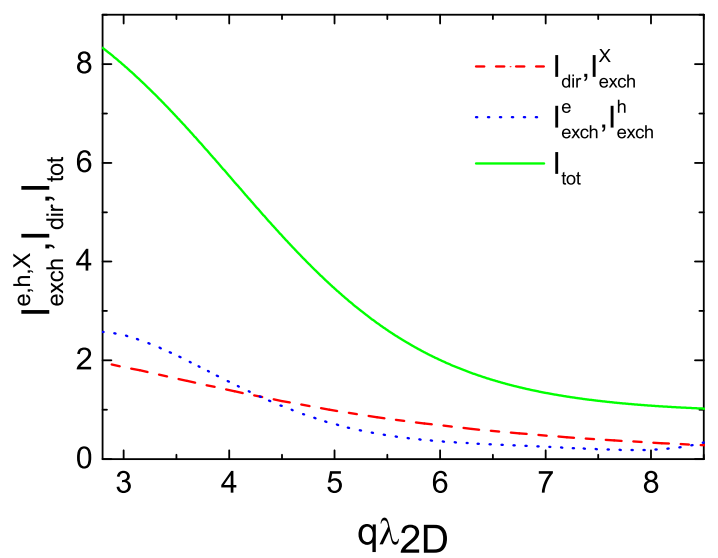

FIG. 2: (Color online) Integrals for electron (hole) and exciton exchange (direct) scattering (interrupted lines), and their sum (solid line). This shows that the strength of the scattering processes is of the same order as compared to the strength of polariton-polariton interaction.

reservoir formation under this scheme, we consider the dynamic of relaxation of the upper branch polaritons at $k=0$ into reservoir excitons at $\pm q$. We must also include the thermal relaxation of these excitons to lower energy states, and therefore introduce a population " $c x$ " of cold excitons. A set of semi-classical Boltzmann equations written for the second- and first-order scattering processes ${ }^{34}$ provide the equation of motion for their populations:

$$
\begin{aligned}
\dot{n}_{0}= & P_{0}-\gamma_{0} n_{0}+\sum_{k} 2 w_{k 0} n_{k} n_{-k}\left(1+n_{0}\right)^{2} \\
& -\sum_{k} 2 w_{0 k} n_{0}^{2}\left(1+n_{k}\right)\left(1+n_{-k}\right), \\
\dot{n}_{ \pm q}= & -\sum_{c x} w_{q c x}\left(1+n_{c x}\right) n_{ \pm q}-w_{q 0} n_{q} n_{-q}\left(1+n_{0}\right)^{2} \\
& +w_{0 q} n_{0}^{2}\left(1+n_{q}\right)\left(1+n_{-q}\right), \\
\dot{n}_{c x}= & \sum_{q} w_{q c x}\left(1+n_{c x}\right) n_{q}-\gamma_{c x} n_{c x} .
\end{aligned}
$$

We have included $\gamma_{0, c x}$ the polariton (exciton) inverse lifetime, $w_{i k}$ are the scattering rates between $i$ and $k$ states, and $P_{0}$ is the pumping rate of the UPB. Since the reservoir excitons lie outside of the light cone, we consider that their lifetime is limited only by their scattering rate $w_{q c x}$ to cold states. Finally, taking into account that $n_{-q}=n_{q}$, the set of equations is reduced to:

$$
\begin{aligned}
& \dot{N}_{0}=P_{0}-\gamma_{0} N_{0}-2 W_{0 R} N_{0}^{2}, \\
& \dot{N}_{R}=-W_{R c x} N_{R}+W_{0 R} N_{0}^{2}, \\
& \dot{N}_{c x}=W_{R c x} N_{R}-\gamma_{c x} N_{c x},
\end{aligned}
$$


where $W_{R c x}\left(W_{0 R}\right)$ is the total scattering rate from the reservoir to cold excitons (from initial state to the reservoir), $N_{0}, N_{R}$ and $N_{c x}$ are the total densities of polaritons, reservoir and cold excitons respectively. The value of $W_{R c x}$ is important since it constrains the attractive reservoir lifetime. From acoustic relaxation only, the effective time $\tau_{R c x}=1 /\left(2 W_{R c x}\right)$ takes values from tens of picoseconds to nanoseconds depending on the $2 \mathrm{D}$ exciton Bohr radius and the width of the quantum well ${ }^{34}$.

For excitons with a small Bohr radius, e.g., in GaAs, although this time, which is tens of picoseconds, is short relatively to the cold exciton radiative lifetime $\tau_{c x}=$ $1 /\left(2 \gamma_{c x}\right)$, it remains long with respect to the polariton lifetime $\tau_{0}=1 /\left(2 \gamma_{0}\right)$, so an attractive reservoir could be obtained for some finite duration of time, e.g., in pulsed excitation experiments. Such conditions occur, for example, in recent experimental work showing the collapse of the wavepacket ${ }^{21}$. For excitons with a large Bohr radius, $\tau_{R c x} \gg \tau_{c x}$ and the attractive potential can be sustained in the CW regime. The total density of excitons in the reservoir is easily obtained from the steady-state of the Boltzmann equations (5):

$$
N_{R s}=\frac{W_{0 R}}{W_{R c x}} N_{0 s}^{2},
$$

while for the population of cold excitons:

$$
N_{c x s}=\frac{W_{R c x}}{\gamma_{c x}} N_{R s}=\frac{W_{0 R}}{\gamma_{c x}} N_{0 s}^{2},
$$

with $N_{0 s}$ the steady-state value of the polariton density:

$$
N_{0 s}=\sqrt{\left(\frac{\gamma_{0}}{4 W_{0 R}}\right)^{2}+\frac{P}{W_{0 R}}}-\frac{\gamma_{0}}{4 W_{0 R}}
$$

Since $W_{0 R}$ has the same order of magnitude as the strength of polariton-polariton interaction (it is possible to get the product $\hbar W_{0 R} N_{0}$ up to meV scale), and the reservoir exciton lifetime is very long $\left(\hbar \gamma_{R}<0.1 \mu \mathrm{eV}\right)$, one can easily obtain the ratio $N_{R s} / N_{0} \gg 1$. Also, if $W_{R c x} \ll \gamma_{c x}$, we can obtain $N_{R s} / N_{c x} \gg 1$, which means that the attractive forces acting on the ground-state polaritons will prevail.

\section{POLARITON DYNAMICS.}

Equations [5] can now be extended to describe the coherent dynamics of the polaritons. We write a set of nonlinear Schrödinger equations for the wavefunction of the upper polaritons $\left(\psi_{0}(\mathbf{r}, t)\right)$ coupled to the attractive exciton reservoir $\left(N_{R}(\mathbf{r}, t)\right)$, which, in turn, are coupled to the gas of repulsive cold excitons $\left(N_{c x}(\mathbf{r}, t)\right)$ :

$$
\begin{aligned}
i \frac{d \psi_{0}(\mathbf{r}, t)}{d t} & =\left[-\frac{\hbar}{2 m_{0 p}} \Delta-i \gamma_{0}+\alpha\left|\psi_{0}(\mathbf{r}, t)\right|^{2}+2 \alpha\left|N_{c x}(\mathbf{r}, t)\right|+\beta\left|N_{R}(\mathbf{r}, t)\right|-i W_{0 R}\left|\psi_{0}(\mathbf{r}, t)\right|^{2}\right] \psi_{0}(\mathbf{r}, t)+P(\mathbf{r}, t) \\
\frac{d N_{R}(\mathbf{r}, t)}{d t} & =\left[-\frac{\hbar}{2 m_{R}} \Delta-W_{R c x}\right] N_{R}(\mathbf{r}, t)+P_{R}(\mathbf{r}, t) \\
\frac{d N_{c x}(\mathbf{r}, t)}{d t} & =-\gamma_{c x} N_{c x}(\mathbf{r}, t)+W_{R c x} N_{R}(\mathbf{r}, t)
\end{aligned}
$$

Here, $\alpha$ and $\beta$ are the polariton-polariton and excitonpolariton interaction constants. As discussed previously, there are different second order processes which could affect the value of $\alpha$, depending on the detuning. However, $\alpha$ can only be changed quantitavely, i.e., its absolute value can change but not its sign. The reduction of $\alpha$ enhances the attraction from the reservoir, so we will consider the worst case, when $\alpha$ is defined only by repulsive interactions. In this case, $\alpha$ is proportional to the product $X^{2} I_{t o t}(\Delta Q=0, q=0)$, while $\beta \sim X I_{\text {tot }}(\Delta Q \neq 0, q=0) . X$ is the Hopfield coefficient which is $1 / 2$ at resonance. Taking $I_{\text {tot }}=-30$ for $\alpha$ and $I_{\text {tot }}=5$ for $\beta$, we obtain a ratio $\alpha / \beta=-3$. The scattering rate $W_{0 R}$ is of the same order as $\beta$. The term $P(\mathbf{r}, t)$, that is responsible for the polariton creation, describes the resonant optical injection and is fixed externally by the experimentalist. We assumed a Gaussian in space for the simulation. The term $P_{R}(\mathbf{r}, t)$ describes the exciton creation and is defined self-consistently by the equation of motion. Its amplitude is proportional to $W_{0 R}\left|\psi_{0}(\mathbf{r}, t)\right|^{4}$, modulated by the Fourier transform of an ellipse in the reciprocal space, as long as excitons are created in excited states.

Excitons have a slow motion as compared to the polaritons, due to their heaviness, and in Eqs. (9) of the main text, we neglect indeed the motion of the cold excitons. However, the group velocity of hot excitons in the region of interest in the reciprocal space $\left(q_{R}=\Delta Q \sim 10^{8} \mathrm{~m}^{-1}\right)$ is about $1 \mu \mathrm{m}$ per $40 \mathrm{ps}$, which remains slow at the scale of the polariton lifetime but should be considered at the timescales over which the reservoir is expected to hold. Therefore, we do not neglect the motion of the reservoir excitons in our calculation. However, the numerical integration of Eq. (9) in this form, i.e., with a Laplacian for the diffusion, is costly in time. To facilitate the computation, we approximate the reservoir exciton motion as 


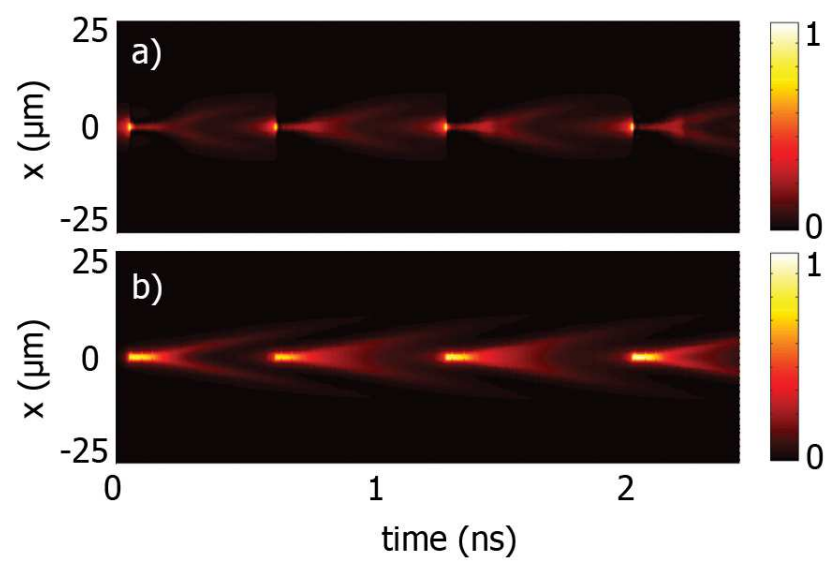

FIG. 3: (Color online) Time evolution of a) the polariton intensity $\left|\psi_{0}\right|^{2}$ and b) the reservoir exciton intensity $\left|N_{R}\right|$. The interplay of attraction and repulsion bring the polariton emission into a self-oscillation regime.

ballistic, i.e., we solve:

$$
\begin{aligned}
& \frac{d N_{R}(\mathbf{r}, t)}{d t}=\frac{\hbar}{2 m_{R}} q_{R}\left|\nabla N_{R}(\mathbf{r}, t)\right|- \\
& -W_{R c x} N_{R}(\mathbf{r}, t)+W_{0 R}\left|\psi_{0}(\mathbf{r}, t)\right|^{4} .
\end{aligned}
$$

In other words, we approximate the exciton dispersion by a linear function, whose slope is tangent to the actual parabolic dispersion at high wavevectors. In this way, we can shift the excitons momentum to the origin of the reciprocal space without changing their motion. This approximation only neglects the diffusion of excitons, which, however, can be neglected just like the motion of the cold excitons. Also, for simplicity we neglect all the possible nonlinear terms in the equation for the exciton motion.

We can now solve Eqs. (9) numerically. We consider a CW coherent resonant excitation at the bottom of the UPB with a Gaussian spatial profile in planar geometry. We take the following parameters: $\tau_{R c x}=2 \mathrm{~ns}$, $\tau_{c x}=200 \mathrm{ps}$, corresponding to an exciton of $30 \mathrm{~nm}$ Bohr radius, and an excitation spot of $3 \mu \mathrm{m}$. The time evolution of the polariton intensity is shown in Fig. 3 a. Here we have considered the upper polariton branch only, while dealing with the lower branch is more convenient and popular in the laboratory due to the fast dephasing time of the UPB in most of currently available samples. Essentially the same physics and ideas apply to lower branch polaritons in the presence of the attractive reservoir, giving qualitatively the same behavior.

It appears right away that the interplay of attraction and repulsion indeed results in a nontrivial dynamics of the polariton intensity, featuring beatings in time with a period of $500 \mathrm{ps}$. This represent a significant improvement on the recent experimental reports of oscil- lating emission from a polariton condensate $\frac{16}{}$, since in our case the oscillation does not relax towards an equilibrium or decays with the condensate, but is sustained forever by the external continuous pumping, representing therefore a stronger case of self-oscillations. This behavior can be easily understood. Polaritons introduced in the cavity are partially converted to reservoir excitons, and the competition between the polariton-polariton repulsion and the exciton-polariton attraction triggers a self-oscillation 26 . When the excitation intensity is strong enough, the attractive interaction prevails, creating a potential dip in the center. Polaritons from the edges of the spot start to flow towards the exciton reservoir, increasing the number of polaritons in the center, and, therefore, also increasing the rate of polariton-exciton conversion. The effect becomes avalanche-like, the polariton wavepacket collapses and the number of excitons in the reservoir increases abruptly, providing a strong redshift for the polariton mode (up to $1.5 \mathrm{meV}$ in our calculations). The energy shift of the ground state makes the optical injection of polaritons inefficient, resulting in a strong diminution of the polariton intensity. The redshift is kept up to $200 \mathrm{ps}$ until the reservoir excitons flow away (their dynamics is shown in Fig. 3b), and the whole dynamics is repeated. The relaxation of reservoir excitons is slow as compared to their flow velocity, and a bunch of repulsive excitons, as well as the repulsive potential that they cause, are created outside of the excitation spot, not affecting the polariton dynamics in its center. The transition of the system from a normal behavior with constant emission intensity to the oscillating regime occurs when the excitation intensity crosses a threshold $P_{t h}$, which could be roughly estimated from Eqs. (46 6). The transition between the two regimes occurs when the inward flow compensates the outwards flow, thus establishing a steady-state in the center of the spot. The value of the treshold $P_{t h}$ is obtained from solving the equation that establishes the threshold $\alpha N_{0 s}+2 \alpha N_{c x s}=-\beta N_{R s}$ along with Eqs. (6), (7) and (8).

\section{CONCLUSIONS.}

We have demonstrated the possibility of providing an effective attractive polariton-polariton interaction mediated by an exciton reservoir. This should allow to investigate a much wider range of dynamical behaviors and exotic phases of the polariton systems. We simulated a simple regime of $\mathrm{CW}$ excitation where self-induced $\mathrm{GHz}$ oscillations are sustained, showing the prospects of this mechanism also for applications, such as clocking or pulsed lasers.

Acknowledgements. DVV would like to thank D. Solnyshkov, N. Gippius and M. Glazov for fruitful discussions. This work was supported by the POLAFLOW ERC starting grant and a RyC contract. 
* Electronic address: dmitrii.vishnevsky@gmail.com

${ }^{1}$ C. Weisbuch, M. Nishioka, A. Ishikawa, and Y. Arakawa, Observation of the coupled exciton-photon mode splitting in a semiconductor quantum microcavity, Phys. Rev. Lett. 69, 3314 (1992)

2 A. Kavokin, J. J. Baumberg, G. Malpuech, and F. P. Laussy, Microcavities, Oxford University Press (2011).

3 J. Kasprzak, M. Richard, S. Kundermann, A. Baas, P. Jeambrun, J. M. J. Keeling, F. M. Marchetti, M. H. Szymaska, R. Andr, J. L. Staehli, V. Savona, P. B. Littlewood, B. Deveaud, and Le Si Dang, Bose-Einstein condensation of exciton polaritons, Nature 443, 409 (2006).

4 A. Amo, J. Lefrère, S. Pigeon, C. Adrados, C. Ciuti, I. Carusotto, R. Houdré, E. Giacobino and A. Bramati, Superfluidity of polaritons in semiconductor microcavities, Nature Physics, 5, 805-810 (2009).

${ }^{5}$ K. G. Lagoudakis, B. Pietka, M. Wouters, R. Andr, and B. Deveaud-Plédran , Coherent Oscillations in an Exciton-Polariton Josephson Junction, Phys. Rev. Lett., 105, 120403 (2010).

${ }^{6}$ P. G. Savvidis, J. J. Baumberg, R. M. Stevenson, M. S. Skolnick, D. M. Whittaker, and J. S. Roberts, AngleResonant Stimulated Polariton Amplifier, Phys. Rev. Lett. 84, 1547 (2000).

7 A. Baas, J.-Ph. Karr, M. Romanelli, A. Bramati, and E. Giacobino, Optical bistability in semiconductor microcavities in the nondegenerate parametric oscillation regime: Analogy with the optical parametric oscillator, Phys. Rev. $B \mathbf{7 0} 161307$ (2004).

8 N. A. Gippius, I. A. Shelykh, D. D. Solnyshkov, S. S. Gavrilov, Y. G. Rubo, A. V. Kavokin, S. G. Tikhodeev, and G. Malpuech, Polarization Multistability of Cavity Polaritons, Phys. Rev. Lett. 98, 236401 (2007).

9 T. K. Paraiso, M. Wouters, Y. Leger, F. Morier-Genoud and B. Deveaud-Pledran, Multistability of a coherent spin ensemble in a semiconductor microcavity, Nature Materials 9, 655 (2010).

10 K. G. Lagoudakis, M.Wouters, M. Richard, A. Baas, I. Carusotto, R. André, Le Si Dang, and B. DeveaudPlédran, Quantized vortices in an exciton-polariton condensate, Nature Physics, 4, 706 (2008).

11 M. Sich, D. N. Krizhanovskii, M. S. Skolnick, A. V. Gorbach, R. Hartley, D. V. Skryabin, E. A. Cerda-Méndez, K. Biermann, R. Hey and P. V. Santos, Observation of bright polariton solitons in a semiconductor microcavity, Nature Photonics, 6, 50-55 (2012).

12 Elizabeth A. Donley, Neil R. Claussen, Simon L. Cornish, Jacob L. Roberts, Eric A. Cornell1, and Carl E. Wieman, Dynamics of collapsing and exploding BoseEinstein condensates, Nature, 412, 295-299 (2001).

13 M. M. Glazov, H. Ouerdane, L. Pilozzi, G. Malpuech, A. V. Kavokin, and A. D'Andrea, Polariton-polariton scattering in microcavities: A microscopic theory, Phys. Rev. B $\mathbf{8 0}$ 155306 (2009).

14 M. Vladimirova, S. Cronenberger, D. Scalbert, K. V. Kavokin, A. Miard, A. Lemaitre, J. Bloch, D. Solnyshkov, G. Malpuech, A. V. Kavokin, Phys. Rev. B 82075301 (2010).

15 C. Adrados, T. C. H. Liew, A. Amo, M. D. Martn, D. Sanvitto, C. Antón, E. Giacobino, A. Kavokin, A. Bramati, and L. Via, Motion of Spin Polariton Bullets in Semiconductor Microcavities, Phys. Rev. Lett 107146402 (2011).
16 Milena De Giorgi, Dario Ballarini, Paolo Cazzato, George Deligeorgis, Simos I. Tsintzos, Zacharias Hatzopoulos, Pavlos G. Savvidis, Giuseppe Gigli, Fabrice P. Laussy, and Daniele Sanvitto, Relaxation Oscillations in the Formation of a Polariton Condensate, in print (2014).

17 S.S. Gavrilov, A.S. Brichkin, A.A. Dorodnyi, S.G. Tikhodeev, N.A. Gippius, V.D. Kulakovskii, Polarization Instability in a Polariton System in Semiconductor Microcavities, JETP Letters 92171 (2010).

18 S. S. Gavrilov, A. S. Brichkin, A. A. Demenev, A. A. Dorodnyy, S. I. Novikov, V. D. Kulakovskii, S. G. Tikhodeev, and N. A. Gippius, Bistability and nonequilibrium transitions in the system of cavity polaritons under nanosecond-long resonant excitation, Phys. Rev. B $\mathbf{8 5}$ 075319 (2012).

19 D. V. Vishnevsky, D. D. Solnyshkov, N. A. Gippius, and G. Malpuech, Multistability of cavity exciton polaritons affected by the thermally generated exciton, Phys. Rev. B 85155328 (2012).

20 M. Wouters, T. K. Paraiso, Y. Leger, R. Cerna, F. MorierGenoud, M. T. Portella-Oberli, and B. Deveaud-Plédran, Influence of a nonradiative reservoir on polariton spin multistability, Phys. Rev. B 87045303 (2013).

21 L. Dominici, D. Ballarini, M. De Giorgi, E. Cancellieri, B. Silva Fernndez, A. Bramati, G. Gigli, F. Laussy, D. Sanvitto, Backjet, shock waves and ring solitons in the quantum pond of a polariton superfluid, arXiv:1309.3083 (2013).

22 A.V. Larionov, V.D. Kulakovskii, S. Hofling, C. Schneider, L. Worschech, and A. Forchel, Polarized Nonequilibrium Bose-Einstein Condensates of Spinor Exciton Polaritons in a Magnetic Field, Phys. Rev. Lett. 105256401 (2010).

${ }^{23}$ V. L. Korenev, Optical orientation of the homogeneous nonequilibrium Bose-Einstein condensate of exciton polaritons, Phys. Rev. B 86035310 (2012).

24 T. C. H. Liew, A. V. Kavokin, and I. A. Shelykh, Optical Circuits Based on Polariton Neurons in Semiconductor Microcavities, Phys. Rev. Lett. 101016402 (2008).

25 M. M. de Lima, Jr, M. van der Poel, P. V. Santos, and J. M. Hvam, Phonon-Induced Polariton Superlattices, Phys. Rev. Lett. 97, 045501 (2006).

26 Alejandro Jenkins, Self-oscillation, Physics Reports 525-2 167-222 (2013).

27 Nicolas Cazier, Xavier Checoury, Laurent-Daniel Haret, and Philippe Boucaud, High-frequency self-induced oscillations in a silicon nanocavity, Optics Express 2111 pp. 13626-13638 (2013)

28 M. Combescot, M. A. Dupertuis, and O. BetbederMatibet, Polariton-polariton scattering: Exact results through a novel approach, Europhys. Lett. 79, 17001 (2007).

29 N. Takemura, S. Trebaol, M. Wouters, M. T. PortellaOberli, and B. Deveaud, Polariton Spinor Interactions in a GaAs-based microcavity, arXiv:1310.6506 (2013).

30 A. V. Sekretenko, S. S. Gavrilov, and V. D. Kulakovskii, Polariton-polariton interactions in microcavities under a resonant 10 to 100 picosecond pulse excitation, Phys. Rev. B 88, 195302 (2013).

31 C. Ciuti, V. Savona, C. Piermarocchi, A. Quattropani and P. Schwendimann, Role of the exchange of carriers in elastic exciton-exciton scattering in quantum wells, Phys. Rev. 
B 587926 (1998)

32 F. Tassone and Y. Yamamoto, Exciton-exciton scattering dynamics in a semiconductor microcavity and stimulated scattering into polaritons, Phys. Rev. B 5910830 (1999).

33 C. Diederichs, J. Tignon, G. Dasbach, C. Ciuti, A. Lematre, J. Bloch, Ph. Roussignol, and C. Delalande, Paramet- ric oscillation in vertical triple microcavities, Nature 440 904 (2006).

34 A. Kavokin and G. Malpuech, Cavity Polaritons, Elsevier, (2003). 